\title{
A wideband optical monitor for a planetary-rotation coating-system
}

\author{
Mark B. Campanelli, Douglas J. Smith \\ Laboratory for Laser Energetics - University of Rochester \\ 250 E. River Rd. \\ Rochester, NY 14623 \\ ph: (716) 2754135 fax: (716) 2731014 \\ mcam@lle.rochester.edu \\ dsmi@1le.rochester.edu
}

\section{Introduction}

A substrate-specific, through-planet, wideband optical coating monitor is being developed to increase production yield and the understanding of physical vapor deposition (PVD) coatings fabricated in the Optical Manufacturing Laboratory at the University of Rochester's Laboratory for Laser Energetics. In-situ wideband optical monitoring of planetary rotation systems allows direct monitoring of large, expensive substrates with complex layering schemes. The optical monitor discussed here is under development for coating several large (e.g., $80.7 \mathrm{x}$ $41.7 \times 9.0 \mathrm{~cm}$ ) polarizers for the National Ignition Facility. Wideband optical monitoring of the production substrates is used in concert with an array of crystal monitors for process control, film parameter evaluation, and error detection with associated design reoptimization.

The geometry of a planetary rotation system, which produces good uniformity across large substrates, makes optical monitoring more difficult. Triggering and timing techniques for data acquisition become key to the process because the optical coating is available only intermittently for monitoring. Failure to properly consider the effects of the system dynamics during data retrieval and processing may result in significant decreases in the spectral data's reliability. Improved data accuracy allows better determination of film thicknesses, indices, and inhomogeneities and enables in-situ error detection for design reoptimization. Techniques using wideband optical monitoring have been described previously in the literature. ${ }^{1-7}$

\section{Description of the system}

The monitor described here is employed on a $1.8 \mathrm{~m}$, electron-beam-gun box-coater (Fig. 1). A counterrotating planetary system precludes central optical monitoring of product-substrates. Deposited coatings typically consist of hafnia-silica dielectric multilayers with a third source used for other materials as needed. Deposition rate is controlled by a quartz crystal monitor/controller, which is one of seven crystal monitors arranged linearly just below the substrate plane and level with the uniformity masks.

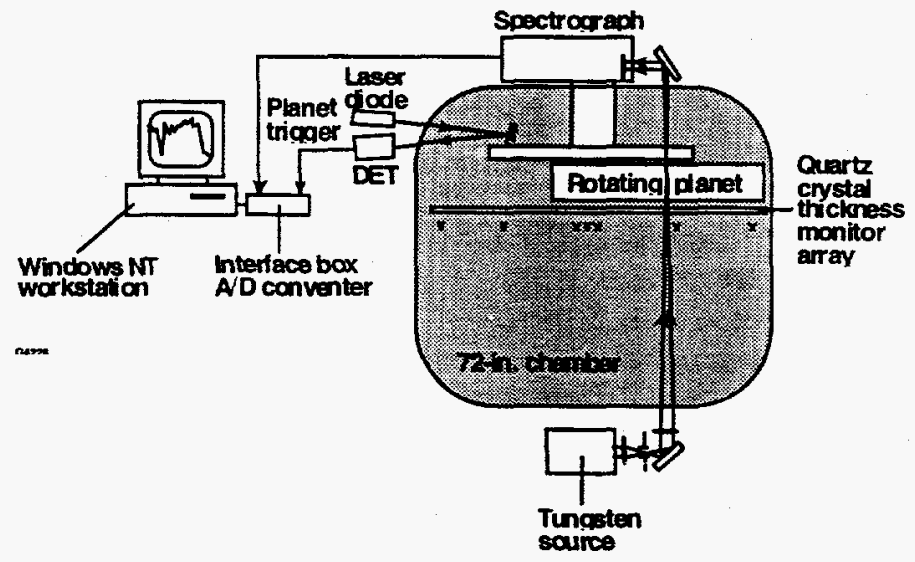

Fig. 1. Diagram of the system.

The optical monitoring system is similar to one described by Li and Yen. ${ }^{5}$ Components of the monitor include a tungsten-halogen source, transport optics, and a flat-field spectrograph for linear dispersion of the spectrum from 350 to $1000 \mathrm{~nm}$ across a 1024 silicon element, EG\&G Reticon S-series linear photodiode array. 


\section{DISCLAIMER}

This report was prepared as an account of work sponsored by an agency of the United States Govermment. Neither the United States Government nor any agency thereof, nor any of their employees, makes any warranty, express or implied, or assumes any legal liability or responsibility for the accuracy, completeness, or usefulness of any information, apparatus, product, or process disclosed, or represents that its use would not infringe privately owned rights. Reference herein to any specific commercial product, process, or service by trade name, trademark, manufacturer, or otherwise does not necessarily constitute or imply its endorsement, recommendation, of favoring by the United States Government or any agency thereof. The views and opinions of authors expressed herein do not necessarily state or reflect those of the United States Government or any agency thereof. 


\section{DISCLAIMER}

Portions of this document may be illegible in electronic image products. Images are produced from the best available original document. 
Information from the crystal monitors and rate controller is integrated with the optical monitor using a PC. This computer will also reoptimize the coating design during deposition when errors are detected and program the deposition controller as necessary. The reoptimization software is being developed separately by A. Tikhonravov at Moscow State University.

\section{Design of the optical monitor}

\section{Triggering and timing considerations}

Timing the acquisition of spectral scans becomes an important consideration when an optical monitor is incorporated with a planetary rotation. The stationary illumination beam from the tungsten-halogen source below the coating chamber is aligned vertically at the radius of rotation of the planetary system (Fig. 1). The planetary tooling is designed so that the rotational hub of each planet has a circular opening $50 \mathrm{~mm}$ in diameter. This opening allows light to pass vertically through the center of each successive planet once per revolution. In addition to the planet holes, a separate $50 \mathrm{~mm}$ hole in the orbiter plate provides a reference signal for normalization throughout the coating process. Vignetting occurs as the planet and reference apertures swing through the beam path. To minimize this effect, the spot diameter of the beam is kept smaller than the diameter of the apertures.

The belt-driven planetary rotation system allows variable rotation speed settings but lacks any inherent rotational tracking capabilities. Consequently, an optical trigger, consisting of a laser diode and a detector, is employed to signal when each hole is coming into alignment with the illumination beam. Tracking the planetary rotation is accomplished by time-analyzing the sequence of electronic pulses that serve as triggers for data acquisition. The timing pattern that develops between successive triggers provides the information necessary for the computer to interpret which spectrum refers to which planet or the reference hole. With knowledge of the system geometry and by assuming a uniform rotational velocity, this method associates an asymmetry in the geometrical distribution of the holes with an asymmetry in the timed sequence of trigger pulses. Once the sequence of holes is determined, the computer continuously acquires spectra through successive planets and the reference hole, preprocesses the data based on its position, and distributes the data appropriately to the reoptimization software.

\section{Data retrieval and processing}

The photodiode array circuitry outputs a continuously updated stream of voltage data corresponding to repeating spectral scans of the light incident on the slit of the spectrograph. For most of the rotation period the light path is completely obstructed by tooling. The spectrograph slit is illuminated for only brief moments. It is the controlling software's function to determine when the data available in the stream correspond to the transmission of light through a passing planet or the reference hole.

One result of this system dynamic is that the intensity of the light when it does reach the detector is not constant, but rather shows an enveloping feature (Fig. 2) that must be accounted for in the signal processing. The profile of this envelope is a function of the velocity of rotation as well as the integration time of the photodiode array and the power of the illumination source. This enveloping on the response curves of the detector must be handled carefully because intensity shifts across successive scans can create a large source of uncertainty in the spectral measurements. After A to D conversion, the software typically selects the spectrum with maximum response, although other averaging schemes are also possible. Normal rotation speeds produce a data-collection window of about $125 \mathrm{~ms}$. With an integration time of $22.16 \mathrm{~ms}$, the photodiode array produces five or six spectral scans per passing hole. Of these spectra, the middle two or three scans in the sequence usually show little variation in light intensity. Scans at the edges of the sequence show a substantial decrease in intensity - evidence of a partially obstructed light path when the aperture rotates into and out of alignment.

Spectral data for all planets and the reference can be updated once per revolution of the planetary system. Because of the size limitations of common storage media, not all data collected are retained for later review. The most current spectral information is always supplied to the reoptimization software, while end-of-layer spectra are archived along with any design changes and special events that occur during the coating run.

\section{Conclusion}

Substrate-specific, through-planet optical monitoring presents an enticing opportunity for expanding both production capability and the understanding of coating processes and film parameters. Optical monitoring of large substrates in planetary rotation, while more challenging than monitoring small, stationary witness pieces, allows 
information on a substrate-specific level to be used in controlling the coating process. The error detection and in-situ reoptimization afforded by through-planet optical monitoring promises to increase production yields. These considerations are even more important because of the expense associated with the numerous large optics being produced for the National Ignition Facility.

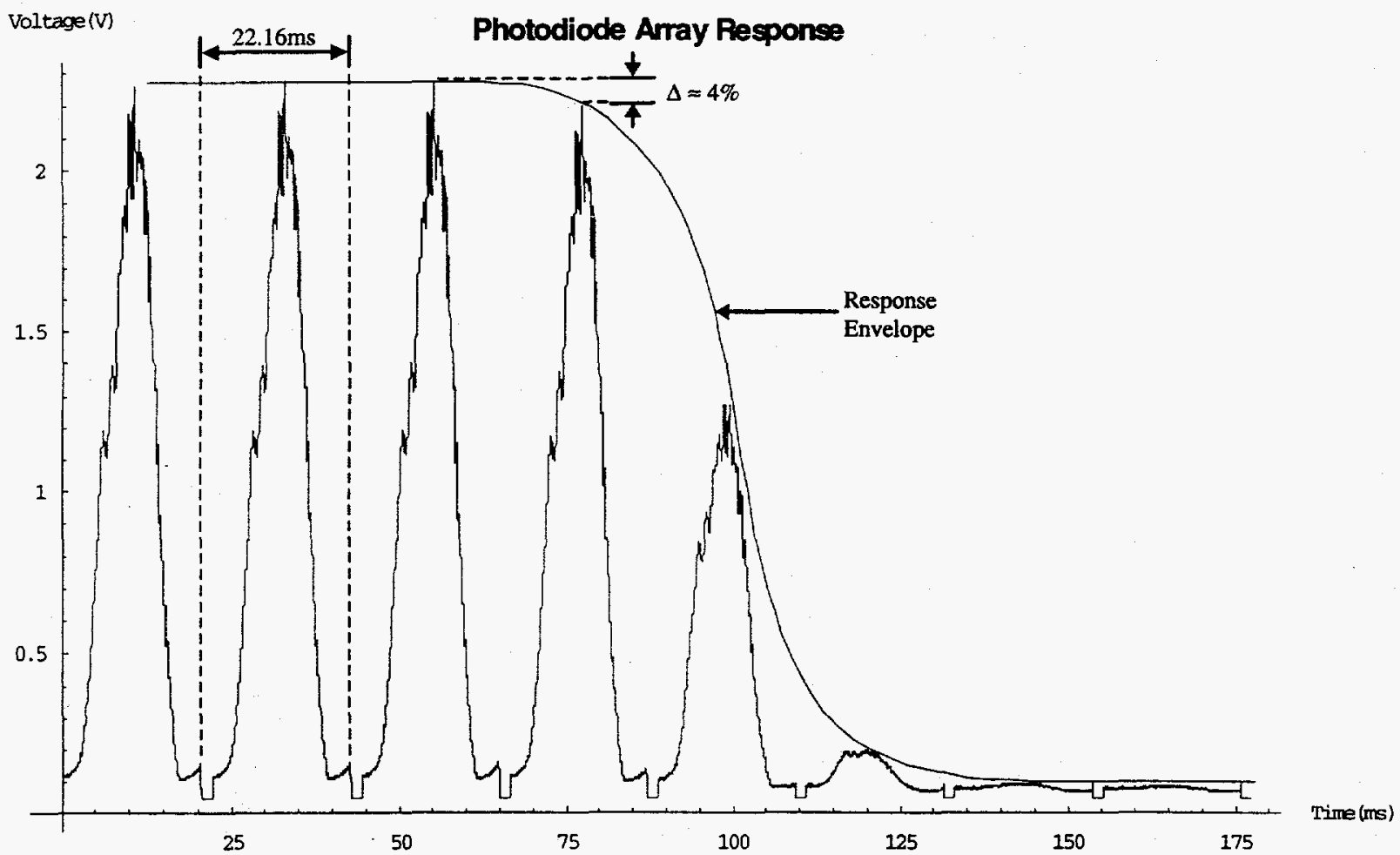

Fig. 2. Evidence of vignetting as a planet moves out of alignment with the illumination beam.

This work was supported by the U.S. Department of Energy Office of Inertial Confinement Fusion under Cooperative Agreement No. DE-FC03-92SF19460, the University of Rochester, and Lawrence Livermore National Laboratory under subcontract B326760. The support of DOE does not constitute an endorsement by DOE of the views expressed in this article.

\section{References}

1. Y.S. Rodichev, "A photometric device for monitoring layer thicknesses during the manufacture of optical coatings in vacuum," Sov. J. Opt. Technol. 42, 398-400 (1975).

2. H. A. Macleod, "Monitoring of optical coatings," Appl. Opt. 20, 82-89 (1981).

3. F. J. Van Milligen, B. Bovard., M. R. Jacobson, J. Mueller, R. Potoff, R. L. Shoemaker, and H. A. Macleod, "Development of an automated scanning monochromator for monitoring thin films," Appl. Opt. 24, 1799-1802 (1985).

4. I. Powell, J. C. M. Zwinkels, and A. R. Robertson, "Development of optical monitor for the control of thin-film deposition," Appl. Opt. 25, 2351-2360 (1986).

5. L. Li and Y.-H. Yen, "Wideband monitoring and measuring system for optical coatings," Appl. Opt. 28, 28892894 (1989).

6. B. T. Sullivan and J. A. Dobrowolski, "Deposition of optical multilayer coatings with automatic error compensation. I. Theoretical description," Appl. Opt. 31, 3821-3835 (1992). And references therein.

7. B. T. Sullivan and J. A. Dobrowolski, "Deposition of optical multilayer coatings with automatic error compensation. II. Experimental results-sputtering system," Appl. Opt. 32, 2351-2360 (1993). 